\title{
Ray tracing with multi-radiation transmitters
}

\author{
R. Brem and T. F. Eibert \\ Lehrstuhl für Hochfrequenztechnik, Technische Universität München, 80290 Munich, Germany \\ Correspondence to: R. Brem (rbrem@tum.de)
}

\begin{abstract}
A restriction in using electromagnetic ray tracing for field prediction is given by the far-field condition: the results are only valid in the far-field region of the radiator. In this paper, it will be shown how ray tracing for accurate field computation can also be applied in the near-field regions of transmitters. The reduction of required large distances between transmitter and receiver is achieved by subdividing the transmitter in smaller subtransmitters. Even for complex transmitters, e.g. antennas with objects in close proximity such as metallic carrier platforms, subtransmitter models can be very efficiently generated by using the Multilevel Fast Multipole Method (MLFMM). This well-known integral equation solving technique makes very large problems in computational electromagnetics manageable. The subtransmitters can be directly generated based on this algorithm. A simulation example will show the improved modeling accuracy and options for simplification and refinement will also be discussed.
\end{abstract}

\section{Introduction}

Electromagnetic ray tracing is a widespread technique for field prediction in large scenarios. It is based on Geometrical Optics (GO), where the assumption is that electromagnetic energy propagates along straight lines. The underlying physical properties of these rays are energy conservation and Fermat's principle. With the extensions Geometrical Theory of Diffraction (GTD) and Uniform Theory of Diffraction (UTD) the diffraction behavior of rays can be explained (Balanis, 1989). Ray tracing finds its use for electrically large problems where exact solvers, e.g. integral equation solvers based on the Method of Moments (MoM) or finite element solvers, would be far too inefficient due to the complexity. A typical example is the use of ray tracing in the design phase of mobile networks. Besides the feasibility of field prediction for such scenarios, further benefits of ray tracing compared to exact field computation methods are the nonexistence of memory and simulation time increase with increasing frequency and an easy parallelization (Cavalcante et al., 2006; Epstein and Rhodes, 2010).

Two approaches exist: in deterministic ray tracing, all rays between transmitter and receiver are exactly computed by image theory. For this, the necessary preprocessing can be quite time-consuming. In ray launching (also called shooting and bouncing rays (SBR) (Ling et al., 1989)), rays are sent out in arbitrary directions where no preprocessing is required. In order to avoid endless running rays which never reach the receiver in ray launching, rays will be neglected after a certain number of interactions or attenuation. Due to the fact that a ray will normally not hit a receive point exactly in ray launching, a tolerance is introduced by using a receiver sphere. Both methods will give the same results if enough rays in the ray launching method are used to cover all relevant paths. Many concepts were introduced to improve the accuracy of ray-tracers (Mohtashami and Shishegar, 2008; Mohtashami et al., 2010) and to speed up the computation (Mohtashami and Shishegar, 2009; Park et al., 2011).

The simplest transmitter model is a localized source with the corresponding far-field radiation pattern. This is an appropriate model when the distance between transmitter and receiver is large enough, i.e. the far-field condition has to be fulfilled. A reduction of this distance increases the error in field computation which can lead to very inaccurate simulation results. For areas of application such as indoor radio channel measurements or car-to-car communication where distances between transmitter and ray-intersecting objects are small or the radiators are large, respectively, this can deteriorate simulation performance. In order to overcome this problem, the transmitter is partitioned in subtransmitters which are also modeled by their far-field radiation patterns.

In the next section, the modeling procedure is presented. A simulation example verifies the better field computation results and an option for model improvement is also shown. 


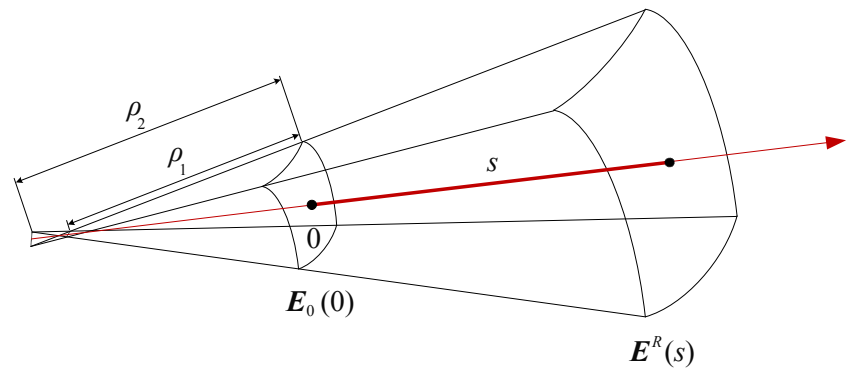

Fig. 1. Astigmatic ray tube.

\section{Procedure}

In general, the electric field $\boldsymbol{E}$ dependent on an electric source current density $\boldsymbol{J}$ can be written according to (Balanis, 1989)

$$
\boldsymbol{E}(\boldsymbol{r})=-j k Z \iint_{V}\left(\overline{\boldsymbol{I}}+\frac{1}{k^{2}} \nabla \nabla\right) \frac{\mathrm{e}^{-j k\left|\boldsymbol{r}-\boldsymbol{r}^{\prime}\right|}}{4 \pi\left|\boldsymbol{r}-\boldsymbol{r}^{\prime}\right|} \cdot \boldsymbol{J}\left(\boldsymbol{r}^{\prime}\right) \mathrm{d} v^{\prime},
$$

where $k=\omega \sqrt{\varepsilon \mu}$ is the wave number and $Z=\sqrt{\mu / \varepsilon}$ is the impedance of the considered homogeneous medium, respectively. Typically, free space is considered so that $Z=377 \Omega$. With far-field assumptions, i.e. for $r=|\boldsymbol{r}| \gg\left|\boldsymbol{r}^{\prime}\right|=r^{\prime}$, the field can be written as

$$
\boldsymbol{E}^{F F}(\boldsymbol{r})=-j k Z \frac{\mathrm{e}^{-j k r}}{4 \pi r}(\hat{\vartheta} \hat{\vartheta}+\hat{\varphi} \hat{\varphi}) \cdot \iiint_{V} \mathrm{e}^{j k \hat{r} \cdot \boldsymbol{r}^{\prime}} \boldsymbol{J}\left(\boldsymbol{r}^{\prime}\right) \mathrm{d} v^{\prime}
$$

where $\hat{r}$ is the unit vector in direction of $\boldsymbol{r}$. Such far-fields describe spherical waves, which can be seen by the term $\mathrm{e}^{-j k r} / 4 \pi r$. Ray tracing can be accomplished by taking these fields as sources, where they are a special form of the more general astigmatic ray representation according to (Balanis, 1989)

$\boldsymbol{E}^{R}(s)=\mathrm{e}^{-j k s} \sqrt{\frac{\rho_{1} \rho_{2}}{\left(\rho_{1}+s\right)\left(\rho_{2}+s\right)}} \boldsymbol{E}_{0}(0)$.

This equation is valid along a straight ray path parameterized by $s . \rho_{1}, \rho_{2}$ are the radii of curvature of the astigmatic ray tube visualized by Fig. 1 .

Equation (1) does not have ray field character, so that ray tracing cannot be applied here. It can be shown that the far-field of a source distribution contains also the complete near-field information. However, it is not possible to access this near-field information by a conventional ray tracing approach. Ray tracing compatible far-field representations which describe the near-field of a radiator are necessary to solve this problem. Important is the fact that the transition from near- to far-field region of a transmitter depends on its dimension, where the typical condition for the far-field is $r>2 D^{2} / \lambda$ (with distance $r$, max. extension $D$ of radiator and wavelength $\lambda$ ) (Balanis, 2005). With smaller radiator dimensions, the far-field starts at a smaller distance. Therefore, a subdivision of the radiating object into smaller subtransmitters is helpful. The far-field assumption for the subtransmitter fields is valid at a smaller distance compared to the complete transmitter, so that ray tracing can be applied. This approach enables a ray based far-field representation of the transmitter's near-field where the validness of this modeling approach for decreasing distances to the transmitter can be guaranteed by decreasing also the subtransmitter dimensions, i.e. using a finer model with more subtransmitters.

The determination of current distributions on electric conducting objects like antennas, is typically the first step in solving radiation problems. Fast integral equation solvers like the efficient MLFMM algorithm are used by default for this. Due to the fact that MLFMM (Chew et al., 2001) is based on a hierarchical subdivision of the object into levels and groups, it is an ideal method to generate the subtransmitter models. The subtransmitters are represented by the groups (cubic boxes) which hold a part of the object. The entire object is enclosed in a hierarchical oct-tree of boxes with different sizes on the different levels. The high efficiency of MLFMM relies on describing the interaction between wellseparated surface currents by a hierarchical plane wave representation of the radiated and received fields instead of computing the interaction of each current with each other current, as done in the classical MoM (Method of Moments). For each group, such a plane wave representation is computed and the field at a receiver group is obtained by appropriate translation operators (Chew et al., 2001).

In order to compute the electromagnetic near- and farfields from a known current distribution, the same efficient hierarchical scheme can be employed (Tzoulis and Eibert, 2005). For all lowest level MLFMM groups (smallest boxes), the computed currents are used to generate a plane wave representation of the corresponding radiated fields (Eibert, 2005). A new subtransmitter of the next higher level is obtained by combining eight subtransmitter boxes on the level below. On the higher level, the new subtransmitter has the double box side length and its source point is in the middle of the eight boxes. The field is computed by an aggregation and interpolation operation of the eight subtransmitter fields. The simple single source point transmitter model is obtained by repeating this procedure until only the box on the highest level remains.

All subtransmitters of one level, representing in sum the entire object, are chosen for a ray tracing simulation. The field samples from each transmitter serve as start values $\boldsymbol{E}_{0}$ in Eq. (3). Different subtransmitter models can be easily generated by choosing the lowest level box size and a certain level. 


\section{Simulation example}

Consider a $\lambda / 2$-dipole, oriented along the $z$-axis in a Cartesian coordinate system. The dipole is placed $5 \mathrm{~cm}$ over the center of a perfectly conducting, quadratic plate of $1 \mathrm{~m}^{2}$ in the $x y$-plane, as shown in Fig. 2a. One could imagine a vehicular antenna modeled by such a configuration, where the plate represents a part of the vehicle which significantly influences the radiation. The dipole is fed in the middle by a $1 \mathrm{GHz}$ voltage source. The electric field shall be computed at different distances $d$ from the antenna. For a meaningful error investigation, receiver planes of $1 \mathrm{~m}^{2}$ providing $N=441$ probe points each, are used. The planes are placed along the line $y=x$ and $z=$ const $=1 \mathrm{~m}$, illustrated by Fig. $2 \mathrm{~b}$.

First, the currents on antenna and plate are computed by an MLFMM integral equation solver. Then, the complex electric field is exactly computed for the receiver planes at distances $d=\{10 \mathrm{~m}, 30 \mathrm{~m}, 50 \mathrm{~m}, 100 \mathrm{~m}, 150 \mathrm{~m}, 200 \mathrm{~m}\}$. The solution is compared to a field computation in the same planes by ray tracing with three different discretizations of the radiator: one, four and nine subtransmitters are used. Fig. 3 shows the average relative error, expressed as

$$
\frac{1}{N} \sum_{i=1}^{N}\left|\frac{E_{\mathrm{RT}}^{i}}{E_{0}^{i}}-1\right|
$$

for the different values of $d$ and the three numbers of transmitters. $E_{\mathrm{RT}}^{i}$ and $E_{0}^{i}$ are the $i$-th complex field value of the ray tracing and the exact (MLFMM) solution, respectively.

The figure shows that a distinctive lower error in field computation near the transmitter is achievable by four and nine subtransmitters compared to the traditional model. For this improvement in accuracy, an increase in simulation time must be accepted. However, the following remarks show that this increase does not emerge as critical problem. When the same number of rays for all transmitters and ray launching are used, the simulation time will only linearly increase with the number of subtransmitters. Due to the fact that the scope of this method is the near-field region of the radiator, the distances the rays have to travel are naturally short, which makes the use of more transmitters less problematic. Furthermore, not many subtransmitters are necessary. With only a small number, the accuracy can be considerably increased.

\section{Model improvement}

An easy way to speed up ray tracing simulations is to disregard subtransmitters whose radiated electromagnetic powers are much smaller than those of others. Large radiators may contain subtransmitters with weak currents and hence also fields so that their contribution to the total field is low. This could be extended to neglect subtransmitters which contribute little in a certain direction of interest.

Important for further improvement of ray tracing accuracy is the choice of the subtransmitter radiation centers. Instead

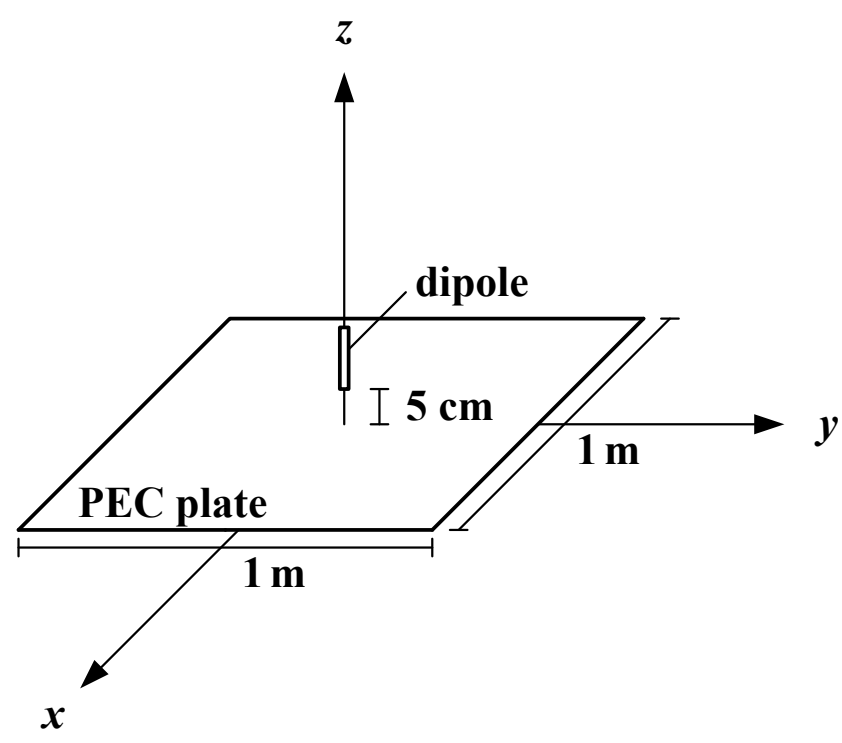

(a) Dipole over PEC plate.

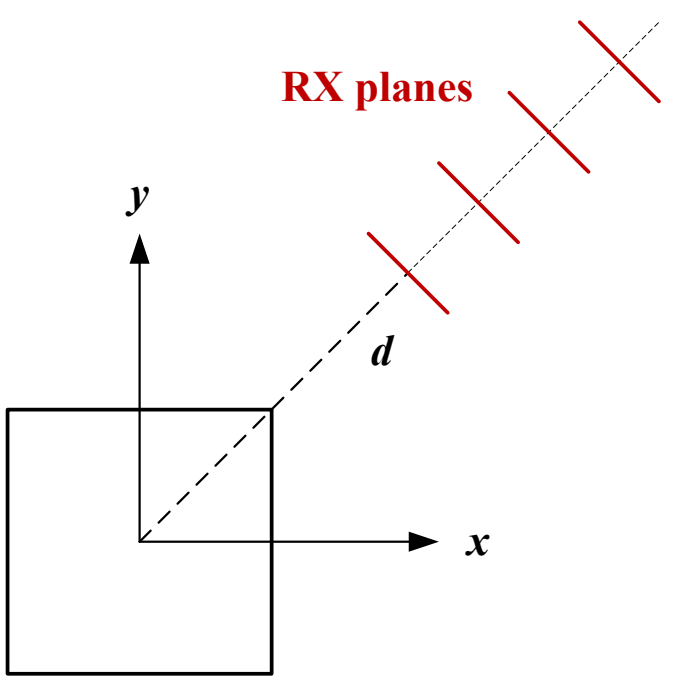

(b) Receiver planes.

Fig. 2. Simulation test.

of using the center of a subtransmitter box, the source point should be positioned at the location of the main current contribution. This position is denoted as current gravity center and computed by

$\boldsymbol{r}=\frac{\sum_{i=1}^{N} w_{i} \boldsymbol{v}_{i}}{\sum_{i=1}^{N} w_{i}} \quad w_{i}=\frac{I_{i}}{\max _{i}\left(I_{i}\right)}$,

where $N$ is the number of current basis functions in the subtransmitter, $\boldsymbol{v}_{i}$ is the edge midpoint of the current basis function $i$ and the weights $w_{i}$ are computed as current magnitude $I_{i}$ over maximum current of the subtransmitter. Prior to a 


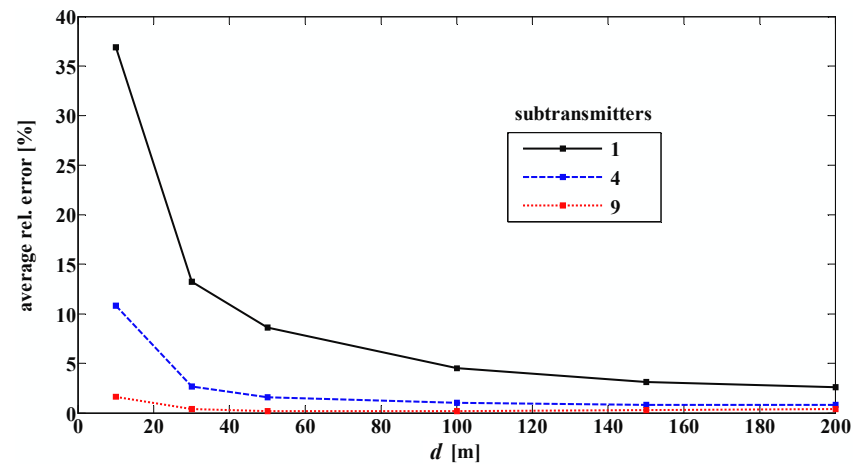

Fig. 3. Average relative error for different numbers of subtransmitters and distances.

ray tracing simulation, the subtransmitter fields are related to these new source points, i.e. the phases must be correctly adapted.

In the simple simulation example of Fig. 2a benefit of these new radiation sources can only be observed for very small distances compared to the values of $d$ due to the small subtransmitters. However, for larger radiating objects a considerable better accuracy for relevant distances can be achieved by this method.

\section{Conclusions}

An approach to produce finer transmitter models for electromagnetic ray tracing was shown. So, accurate field prediction in the near-field region of radio transmitters is made possible. By utilization of the Multilevel Fast Multipole Method, the subtransmitters can be very efficiently generated. Reason for this is the inherent hierarchical subdivision of a radiating object as basic principle of the algorithm. By adjusting the subtransmitter box size on the lowest level or choosing a certain level, great freedom in transmitter model generation exists. The simulation result shows the quality of this method.

\section{References}

Balanis, C.: Advanced Engineering Electromagnetics, John Wiley \& Sons, New York, 1989.

Balanis, C.: Antenna Theory, John Wiley \& Sons, Hoboken, 2005.

Cavalcante, A. M., de Sousa, M. J., Costa, J. C. W., Frances, C. R. L., Protasio dos Santos Cavalcante, G., and de Souza Sales, C.: 3D ray-tracing parallel model for radio-propagation prediction, in: International Telecommunications Symposium, 269274, doi:10.1109/ITS.2006.4433282, 2006.

Chew, W., Jin, J., Michielssen, E., and Song, J.: Fast and Efficient Algorithms in Computational Electromagnetics, Artech House, Boston, MA, 2001.

Eibert, T. F.: A diagonalized multilevel fast multipole method with spherical harmonics expansion of the k-space Integrals, IEEE Trans. Antennas Propag., 53, 814-817, doi:10.1109/TAP.2004. 841310, 2005.

Epstein, B. R. and Rhodes, D. L.: GPU-accelerated ray tracing for electromagnetic propagation analysis, in: IEEE International Conference on Wireless Information Technology and Systems (ICWITS), 1-4, doi:10.1109/ICWITS.2010.5611922, 2010.

Ling, H., Chou, R.-C., and Lee, S.-W.: Shooting and bouncing rays: calculating the RCS of an arbitrarily shaped cavity, IEEE Trans. Antennas Propag., 37, 194-205, doi:10.1109/8.18706, 1989.

Mohtashami, V. and Shishegar, A. A.: A new double-counting cancellation technique for ray tracing using separation angle distribution, in: IEEE International RF and Microwave Conference (RFM), 306-310, doi:10.1109/RFM.2008.4897435, 2008.

Mohtashami, V. and Shishegar, A. A.: Application of wavefront decomposition to ray tube tracing, in: First Conference on Millimeter-Wave and Terahertz Technologies (MMWaTT), 5-9, doi:10.1109/MMWATT.2009.5450465, 2009.

Mohtashami, V., Parsa, S., and Shishegar, A. A.: Efficient enhancement of the accuracy of Ray tracing, in: IEEE Antenn. Prop., 1-4, doi:10.1109/APS.2010.5560920, 2010.

Park, H.-G., Kim, H.-T., and Kim, K.-T.: Beam tracing for fast RCS prediction of electrically large targets, PIER, 20, 29-42, doi:10.2528/PIERM11060702, 2011.

Tzoulis, A. and Eibert, T. F.: Efficient electromagnetic near-field computation by the multilevel fast multipole method employing mixed near-field/far-field translations, IEEE Antenn. Wirel. Pr., 4, 449-452, doi:10.1109/LAWP.2005.860195, 2005. 